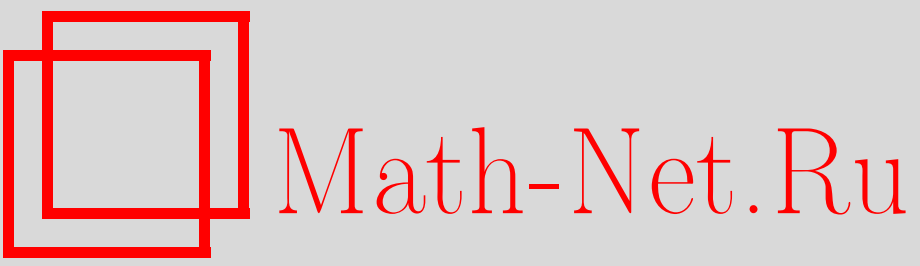

С. В. Нагаев, Теорема восстановления при отсутствии степенных моментов, Теория вероятн. и ее примен., 2011, том 56, выпуск 1, 188-197

DOI: https://doi.org/10.4213/tvp4335

Использование Общероссийского математического портала Math-Net.Ru подразумевает, что вы прочитали и согласны с пользовательским соглашением

http://www.mathnet.ru/rus/agreement

Параметры загрузки:

IP : 54.197 .217 .227

26 апреля 2023 г., 18:04:14 
24. Ламперти Дж. Вероятность. М.: Наука, 1973, 183 с.

25. Питербарг В. И. Асимптотические методы в теории гауссовских случайных процессов и полей. М.: Изд-во Моск. ун-та, 1988, 175 с.

26. Slepian D. The one-sided barrier problem for Gaussian noise. - Bell System Tech. J., 1962 , v. 41, № 2, p. 463-501.

27. Piterbarg V. I., Stamatovich S. On maximum of Gaussian non-centered fields indexed on smooth manifolds. - Asymptotic Methods in Probability and Statistics with Applications. Ed. by N. Balakrishnan, I. A. Ibragimov, and V. B. Nevzorov. Boston: Birkhäuser, 2001, p. 189-203.

28. Konakov V., Piterbarg V. High level excursions of Gaussian fields and the weakly optimal choice of the smoothing parameter. I. - Math. Methods Statist., 1995, v. 4, № 4, p. 421-434.

Поступила в редакцию 6.V.2009

Исправленный вариант 22.II. 2010

(C) 2011 г.

НАГАЕВ C. B.*

\section{ТЕОРЕМА ВОССТАНОВЛЕНИЯ ПРИ ОТСУТСТВИИ СТЕПЕННЫХ МОМЕНТОВ ${ }^{1)}$}

Доказывается теорема восстановления для случайного блуждания, порожденного последовательностью сумм независимых одинаково распределенных случайных величин, распределения которых медленно убывают на бесконечности.

В теории восстановления этот случай ранее не рассматривался.

Ключевые слова и фразы: абелева теорема, вероятность восстановления, медленно меняющаяся функция, представление Караматы, разложение Тейлора, рекуррентный процесс, тауберова теорема.

1. Введение. Пусть $X$ - случайная величина, принимающая целые неотрицательные значения с вероятностями $p_{n}=\mathbf{P}(X=n)$.

Рассмотрим последовательность $X_{1}, X_{2}, \ldots, X_{n}, \ldots$ независимых одинаково распределенных случайных величин, совпадающих по распределению с $X$. Обозначим $S_{n}=\sum_{j=1}^{n} X_{j}$. Вероятность восстановления $h_{n}$ определяется равенством

$$
h_{n}=\sum_{k=0}^{\infty} \mathbf{P}\left(S_{k}=n\right), \quad n \geqslant 0,
$$

если положить $S_{0}=0$. На языке анализа

$$
h_{n}=C_{n}\left(\frac{1}{1-f(z)}\right),
$$

где $f(z)=\sum_{n=0}^{\infty} p_{n} z^{n}$. Символ $C_{n}$ означает $n$-й коэффициент в разложении Тейлора.

* Институт математики им. С.Л. Соболева, пр-т Академика Коптюга, 4, 630090 Новосибирск, Россия; e-mail: nagaev@math.nsc.ru, nagaevs@hotmail.com, nagaevs@academ.org

1) Работа выполнена при поддержке Российского фонда фундаментальных исследований (грант № 09-01-00048-а). 
Согласно классической теории восстановления имеет место альтернатива:

$$
\lim _{n \rightarrow \infty} h_{n}= \begin{cases}(\mathbf{E} X)^{-1}, & \text { если } \mathbf{E} X<\infty, \\ 0, & \text { если } \mathbf{E} X=\infty\end{cases}
$$

(см., например, [1]-[3]).

Естественно, в случае $\mathbf{E} X=\infty$ возникает задача связать скорость убывания $h_{n}$ со скоростью убывания $\mathbf{P}(X>n)$. А. Гарсия и Дж. Ламперти [6] доказали, что если

$$
\mathbf{P}(X>n) \sim \frac{L(n)}{n^{\alpha}}, \quad \frac{1}{2}<\alpha<1,
$$

где $L(n)$ - медленно меняющаяся функция, то

$$
h_{n} \sim \frac{c(\alpha)}{n^{1-\alpha} L(n)}
$$

где $c(\alpha)=\pi^{-1} \sin \pi \alpha$.

Отношение $a_{n} \sim b_{n}$ здесь и ниже означает, что $\lim _{n \rightarrow \infty} a_{n} / b_{n}=1$.

Пусть $\alpha=1 / 2$. Тогда $n^{1-\alpha}=n^{\alpha}$. Следовательно,

$$
\frac{c(\alpha)}{n^{1-\alpha} L(n)}=o(\mathbf{P}(X>n))
$$

если $\lim _{n \rightarrow \infty} L(n)=\infty$. В этом случае не исключено, что для некоторой последовательности $n_{k} \rightarrow \infty$

$$
\frac{c(\alpha)}{n_{k}^{1-\alpha} L\left(n_{k}\right)}=o\left(\mathbf{P}\left(X=n_{k}\right)\right)
$$

Действительно, пусть $F_{1}(x)$ - любая функция распределения, сосредоточенная на множестве целых неотрицательных чисел и удовлетворяющая условию

$$
1-F_{1}(x) \sim \frac{\ln x}{x^{1 / 2}} .
$$

Далее, пусть $F_{2}(x)$ - ступенчатая функция распределения со скачками величины $x_{k}^{-1 / 2}$ в точках $x_{k}=2^{k}$. Нетрудно видеть, что

$$
1-F_{2}\left(x_{k}\right) \sim \frac{\sqrt{2}}{\sqrt{2}-1} x_{k}^{-1 / 2} .
$$

Положим $F(x)=\left(F_{1}(x)+F_{2}(x)\right) / 2$. Нетрудно показать, что при $x \rightarrow \infty$

$$
1-F(x) \sim \frac{\ln x}{2 x^{1 / 2}} .
$$

Кроме того, $F\left(x_{k}+\right)-F\left(x_{k}\right)>\left(F_{2}\left(x_{k}+\right)-F_{2}\left(x_{k}\right)\right) / 2 \sim x_{k}^{-1 / 2} / 2$. Если случайная величина $X$ имеет функцию распределения $F(x)$, определенную выше, то

$$
\frac{2 c(1 / 2)}{x_{k}^{1 / 2} \ln x_{k}}=o\left(\mathbf{P}\left(X=x_{k}\right)\right) .
$$

Это означает, в силу (1.1), что асимптотическое равенство

$$
h_{n} \sim \frac{2 c(\alpha)}{n^{1 / 2} \ln n}
$$

не может выполняться. Приведенный контрпример является модификацией примера 5-А из работы [7].

В случае $0<\alpha<1 / 2$ нетрудно построить аналогичные контпримеры. В качестве $F_{1}(x)$ нужно взять функцию, удовлетворяющую условию $1-F_{1}(n) \sim L(n) / n^{\alpha}$, а $F_{2}(x)$ можно оставить прежней. 
Таким образом, условие (1.3) при $0<\alpha \leqslant 1 / 2$ недостаточно для (1.4). Нужны еще какие-то ограничения на флуктуации вероятностей $p_{n}$. Одно из условий такого рода было предложено в [5], а именно,

$$
p_{n+1} p_{n-1}>p_{n}^{2}
$$

т.е. последовательность $\ln p_{n}$ является выпуклой. Заметим прежде всего, что (1.5) можно записать в виде $p_{n-1} / p_{n}>p_{n} / p_{n+1}$. Таким образом, последовательность $p_{n} / p_{n+1}$ не возрастает, и, следовательно, существует конечный предел $\lambda=$ $\lim _{n \rightarrow \infty} p_{n} / p_{n+1}$. Если $p_{n_{0}} p_{n_{0}+1}^{-1}<\rho<1$ при некотором $n_{0}$, то $p_{n} p_{n+1}^{-1}<\rho$ для всех $n>n_{0}$. Следовательно, $p_{n}>\rho^{n_{0}-n}$, что невозможно. Значит, $p_{n} p_{n-1}^{-1}>1$ для всех $n$. Отсюда, в частности, следует, что $\lambda \geqslant 1$.

Если $\lambda>1$, то $p_{n}<\lambda^{-n}$, но это невозможно, если выполнено условие (1.3).

Таким образом, если одновременно выполнены условия (1.3) и (1.5), то $\lambda=1$. Заметим также, что последовательность $p_{n}$ убывает, поскольку, как мы показали выше, $p_{n} / p_{n+1}>1$.

В [7] доказано (см. следствие 3-A), что (1.4) имеет место при $0<\alpha<1 / 2$, если выполняется (1.3) и $p_{n} \geqslant p_{n+1}$, начиная с некоторого значения $n$. Мы покажем ниже (см. п. 4), что при этом условии

$$
\lim _{n \rightarrow \infty} p_{n} n^{\alpha+1} L^{-1}(n)=\alpha .
$$

Соотношение (1.4) выводится в [6] посредством прямого асимптотического анализа интеграла

$$
\frac{1}{2 \pi} \int_{-\pi}^{\pi} e^{-i n t}(1-f(t))^{-1} d t
$$

который по определению равен правой части формулы (1.2). В [7] рассматривается более общий случай блуждания в $d$-мерном пространстве, $d \geqslant 1$. При этом используется локальная теорема Рвачевой [8].

В настоящей работе исследуется крайний случай, когда $\mathbf{P}(X>n)$ медленно меняется. Основной результат формулируется следующим образом.

Теорема 1.1. Пусть

$$
p_{n} \sim l(n) n^{-1},
$$

где $l(x)$ медленно меняется при $x \rightarrow \infty$. Тогда

$$
h_{n} \sim \frac{\mathbf{P}(X=n)}{[\mathbf{P}(X \geqslant n)]^{2}} .
$$

Согласно лемме 2.2 , из условия (1.7) следует, что функция $\mathbf{P}(X>x)$ медленно меняется. Важно отметить, что (1.8) может не выполняться, если потребовать только, чтобы функция $\mathbf{P}(X>x)$ медленно менялась (см. ниже пример 4.1).

Формула (1.4) допускает простое эвристическое объяснение. Пусть $H(x)-$ функция восстановления, т.е. $H(x)=\sum_{k=0}^{\infty} F^{k *}(x)$. Согласно лемме 2.5 ,

$$
H(k+) \sim \frac{1}{L(k)} .
$$

Отсюда

$$
h_{k}=H(k+)-H(k) \sim \frac{1}{L(k+1)}-\frac{1}{L(k)} \sim \frac{L(k)-L(k+1)}{L^{2}(k)}=\frac{\mathbf{P}(X=k)}{[\mathbf{P}(X \geqslant k)]^{2}} .
$$

Правдоподобное допущение в этих рассуждениях заключается в том, что

$$
H(k+)-H(k) \sim \frac{1}{L(k+1)}-\frac{1}{L(k)} .
$$


Однако в конечном счете оно оказывается верным. Доказательство этого факта и составляет содержание теоремы 1.1.

Если выполнено (1.6), то формулу (1.4) можно записать в виде (1.8) с дополнительным множителем, а именно,

$$
h_{n} \sim c_{1}(\alpha) \frac{\mathbf{P}(X=n)}{[\mathbf{P}(X \geqslant n)]^{2}},
$$

где $c_{1}(\alpha)=(\pi \alpha)^{-1} \sin \pi \alpha$. Очевидно, что $c_{1}(\alpha) \rightarrow 1$ при $\alpha \rightarrow 0$. Поэтому (1.8) можно рассматривать как предельный случай по отношению к (1.9). Далее, согласно (1.6), $n p_{n} \sim \alpha \mathbf{P}(X \geqslant n)$. Следовательно, можно ожидать, что в условиях теоремы 1.1 $n p_{n}=o(\mathbf{P}(X \geqslant n))$, что и подтверждается леммой 2.1 .

Метод доказательства (1.8) кардинально отличается как от аналитического метода работы [6], так и от вероятностного подхода, используемого в [7]. Отметим, что последний не может быть применен в рассматриваемом случае из-за отсутствия локальной предельной теоремы. Что касается интегральной предельной теоремы, то она была доказана еще в 1952 г. в пионерской работе Д. Дарлинга [9]. Недавно эта теорема была передоказана в [10].

В оставшейся части работы будем мы использовать для $\mathbf{P}(X \geqslant x)$ обозначение $L(x)$.

2. Вспомогательные результаты. Мы начнем со следующего утверждения.

Лемма 2.1. Справедливо соотношение

$$
\lim _{x \rightarrow \infty} \frac{l(x)}{L(x)}=0
$$

Д о к а з а т е л ь с т в о. Очевидно, что для любого $c>1$

$$
\mathbf{P}(X \geqslant x)=\sum_{k \geqslant x} l(k) k^{-1}>\min _{c x>k \geqslant x} l(k) \sum_{c x>k \geqslant x} \frac{1}{k} .
$$

Далее, при $x>1, c>2$

$$
\sum_{c x>k \geqslant x} \frac{1}{k}>\int_{x+1}^{c x} y^{-1} d y \geqslant \ln \frac{c x}{x+1}>\ln \frac{c}{2} .
$$

С другой стороны, при любом фиксированном $c>1$

$$
\lim _{x \rightarrow \infty} l^{-1}(x) \min _{x \leqslant k<c x} l(k)=1 .
$$

Таким образом,

$$
\liminf _{x \rightarrow \infty} \frac{L(x)}{l(x)}>\ln \frac{c}{2}
$$

каково бы ни было $c>2$. Следовательно, $\lim _{x \rightarrow \infty} L(x) / l(x)=\infty$. Лемма доказана.

Лемма 2.2. Если выполнено условие (1.7), то функция $L(x)$ медленно меняется при $x \rightarrow \infty$.

Д о к а з а т е л ь с т в о. Очевидно, что для любого $c>1$

$$
\frac{L(c x)}{L(x)}=1+\frac{1}{L(x)} \sum_{x \leqslant k<c x} k^{-1} l(k) .
$$

Далее,

$$
\sum_{x \leqslant k<c x} k^{-1} l(k)<\max _{x \leqslant k<c x} l(k) \sum_{x \leqslant k<c x} k^{-1} .
$$


Нетрудно видеть, что $\lim _{x \rightarrow \infty} l^{-1}(x) \max _{x \leqslant k<c x} l(k)=1$. С другой стороны, $\sum_{x \leqslant k<c x} k^{-1}<\ln c$ при $c>1$. Таким образом, для любого $c>1$

$$
\limsup _{x \rightarrow \infty} \frac{1}{l(x)} \sum_{x \leqslant k<c x} k^{-1} l(k)<\ln c .
$$

Из (2.1)-(2.3) следует утверждение леммы.

Лемма 2.3. Справедливо тождество

$$
n h_{n}=\sum_{k=0}^{n-1}(n-k) p_{n-k} h_{k}^{(2)}
$$

где $h_{n}^{(2)}=\sum_{k=0}^{n} h_{n-k} h_{k}$.

Д о к а з а т е л ь с т в о. Обозначим $h(z)=1 /(1-f(z))$. Очевидно, что

$$
h^{\prime}(z)=\frac{f^{\prime}(z)}{(1-f(z))^{2}} .
$$

Далее,

$$
C_{n}\left(\frac{1}{(1-f(z))^{2}}\right)=h_{n}^{(2)}, \quad C_{n-1}\left(f^{\prime}(z)\right)=n p_{n} .
$$

Отсюда

$$
C_{n}\left(\frac{f^{\prime}(z)}{(1-f(z))^{2}}\right)=\sum_{k=0}^{n}(n-k+1) p_{n-k+1} h_{k}^{(2)} .
$$

С другой стороны,

$$
C_{n-1}\left(h^{\prime}(z)\right)=n h_{n} .
$$

Из (2.5)-(2.7) следует (2.4). Лемма доказана.

Лемма 2.4. При $n \rightarrow \infty$

$$
\sum_{k=0}^{n} L(k) \sim n L(n)
$$

Д о к а з а т е л ь с т в о. Для $L(x)$ имеет место представление Караматы

$$
L(x)=a(x) \exp \left\{\int_{1}^{x} \frac{\varepsilon(u)}{u} d u\right\}, \quad x>1,
$$

где $\lim _{x \rightarrow \infty} \varepsilon(x)=0, \lim _{x \rightarrow \infty} a(x)=a, 0<a<\infty$. Поскольку $\lim _{n \rightarrow \infty} \sum_{k=0}^{n} L(k)=\infty$, то

$$
\sum_{k=0}^{n} L(k) \sim \sum_{x=0}^{n} \tilde{L}(k)
$$

где $\widetilde{L}(x)=a \exp \left\{\int_{1}^{x} u^{-1} \varepsilon(u) d u\right\}$. Нетрудно видеть, что

$$
\sum_{k=0}^{n} \widetilde{L}(k)=\widetilde{L}(n)(n+1)+\sum_{k=1}^{n}(\widetilde{L}(k-1)-\widetilde{L}(k)) k .
$$

Далее, при $n \rightarrow \infty$

$$
\widetilde{L}(k)-\widetilde{L}(k-1)=O\left(\widetilde{L}(k)\left|\int_{k-1}^{k} \frac{\varepsilon(u)}{u} d u\right|\right)=o\left(\frac{\tilde{L}(k)}{k}\right) .
$$

Отсюда

$$
\sum_{k=o}^{n}(\widetilde{L}(k)-\widetilde{L}(k-1)) k=o\left(\sum_{k=0}^{n} \widetilde{L}(k)\right) .
$$

Из (2.9)-(2.11) следует утверждение леммы. 
Лемма 2.5. При $n \rightarrow \infty$

$$
\sum_{k=1}^{n} h_{k} \sim \frac{1}{L(n)}
$$

Д о к а з а т е л ь с т в о. Прежде всего,

$$
\sum_{k=0}^{\infty} \mathbf{P}(X>k) z^{k}=\frac{1-f(z)}{1-z} .
$$

Поскольку $\mathbf{P}(X>k) \sim L(k)$, то, используя лемму 2.4 и абелеву теорему (см. [4, гл. XIII, § 5, теорема 5]), имеем

$$
\frac{1-f(z)}{1-z} \sim(1-z)^{-1} L\left((1-z)^{-1}\right), \quad z \rightarrow 1-.
$$

Отсюда $(1-z)(1-f(z))^{-1} \sim(1-z) L^{-1}\left((1-z)^{-1}\right), z \rightarrow 1-$, т.е.

$$
(1-f(z))^{-1} \sim L^{-1}\left((1-z)^{-1}\right)
$$

Применяя тауберову теорему (она содержится в упомянутой выше теореме 5), мы получаем желаемый результат.

Лемма 2.6. При $n \rightarrow \infty$

$$
\sum_{k=1}^{n} h_{k}^{(2)} \sim \frac{1}{L^{2}(n)} .
$$

Д о к а з а т е ль с т в о. В силу (2.13)

$$
\frac{1}{(1-f(z))^{2}} \sim L^{-2}\left((1-z)^{-1}\right), \quad z \rightarrow 1-.
$$

С другой стороны, как уже отмечалось в доказательстве леммы 2.3 ,

$$
h_{n}^{(2)}=C_{n}\left(\frac{1}{(1-f(z))^{2}}\right) .
$$

Применяя теперь тауберову теорему (см. ссылку в доказательстве леммы 2.5), мы получаем желаемый результат.

Лемма 2.7. При $n \rightarrow \infty$

$$
\sum_{k=1}^{n} l(k) \sim n l(n) .
$$

Д о к а $з$ а т е л ь с т в о. Поскольку при $n \rightarrow \infty$

$$
\sum_{k=1}^{n} l(k)=\sum_{k=1}^{n} k p_{k} \rightarrow \infty,
$$

то в данном случае проходят те же рассуждения, что и при доказательстве леммы 2.4 .

Лемма 2.8. Существует последовательность $\theta_{n}$ такая, что $0 \leqslant \lim \sup _{n \rightarrow \infty} \theta_{n} \leqslant 2 u$

$$
h_{n}^{(2)} \leqslant \theta_{n} L^{-1}(n) \max _{n / 2 \leqslant k \leqslant n} h_{k} .
$$

Д о к а з а т е л ь с т в о. Нетрудно видеть, что

$$
h_{n}^{(2)} \leqslant 2 \sum_{0 \leqslant k \leqslant n / 2} h_{k} h_{n-k} \leqslant 2 \max _{n / 2 \leqslant k \leqslant n} h_{k} \sum_{0 \leqslant k \leqslant n / 2} h_{k} .
$$

Чтобы завершить доказательство, остается применить лемму 2.5 . 
Лемма 2.9. Существуют положительнье последовательности $a_{n}$ и $b_{n}$ такие, umo $\lim _{n \rightarrow \infty} a_{n}=1,0<\lim \sup _{n \rightarrow \infty} b_{n} \leqslant 2 u$

$$
h_{n}=a_{n} \frac{l(n)}{n L^{2}(n)}+b_{n} \frac{l(n)}{L(n)} \max _{n / 2<k \leqslant n} h_{k} .
$$

Д о к а з а т е л ь с т в о. Согласно лемме 2.3 ,

$$
n h_{n}=\sum_{0 \leqslant k \leqslant n / 2}(n-k) p_{n-k} h_{k}^{(2)}+\sum_{n / 2<k \leqslant n-1}(n-k) p_{n-k} h_{k}^{(2)} \equiv \sum_{1}+\sum_{2} .
$$

Используя леммы 2.6 и 2.7 , имеем

$$
\sum_{1}=\sum_{0 \leqslant k \leqslant n / 2} l(n-k) h_{k}^{(2)} \sim l(n) \sum_{0 \leqslant k \leqslant n / 2} h_{k}^{(2)} \sim \frac{l(n)}{L^{2}(n)} .
$$

С другой стороны, в силу лемм 2.7 и 2.8 ,

$$
\begin{aligned}
\sum_{2}= & \sum_{n / 2<k \leqslant n-1} l(n-k) h_{k}^{(2)} \leqslant \theta_{n} \max _{n / 2<k \leqslant n} h_{k} L^{-1}(n) \\
& \times \sum_{0 \leqslant k \leqslant n-1} l(n-k) \sim \theta_{n} n \max _{n / 2<k \leqslant n} h_{k} l(n) L^{-1}(n) .
\end{aligned}
$$

Комбинируя полученные соотношения, мы приходим к утверждению леммы.

3. Доказательство основного результата. Отправной точкой является равенство (2.15). Полагая для краткости

$$
\varphi(n)=a_{n} \frac{l(n)}{n L^{2}(n)}, \quad \psi(n)=b_{n} \frac{l(n)}{L(n)},
$$

запишем (2.15) в виде

$$
h_{n}=\varphi(n)+\psi(n) \max _{n / 2<k \leqslant n} h_{k} .
$$

Зафиксируем $0<\varepsilon<1 / 2$ и выберем $N$ так, чтобы выполнялось неравенство $\psi(n)<\varepsilon$ для $n>N$. Пусть $n_{1}$ - значение $k$, при котором достигается $\max _{n / 2<k \leqslant n} h_{k}$. В частности, может случиться, что $n_{1}=n$. В этом случае $h_{n}<\varphi(n) /(1-\varepsilon)$. Если $N<n_{1}<n$, то

и, следовательно,

$$
h_{n_{1}}<\varphi\left(n_{1}\right)+\varepsilon \max _{n_{1} / 2 \leqslant j \leqslant n_{1}} h_{j}
$$

$$
h_{n}<\varphi(n)+\varepsilon \varphi\left(n_{1}\right)+\varepsilon^{2} \max _{n_{1} / 2<j \leqslant n_{1}} h_{j} .
$$

Если $\max _{n_{1} / 2<j \leqslant n_{1}} h_{j}=h_{n_{1}}$, то $h_{n_{1}}<\varphi\left(n_{1}\right) /(1-\varepsilon)$. Подставляя эту оценку в $(3.2)$, имеем

$$
h_{n}<\varphi(n)+\varepsilon \varphi\left(n_{1}\right)+\frac{\varepsilon^{2}}{1-\varepsilon} \varphi\left(n_{1}\right) .
$$

Если $\max _{n_{1} / 2<j \leqslant n_{1}} h_{j}$ достигается для $N<j<n_{1}$, то точно так же получим неравенство

$$
h_{n}<\varphi(n)+\varepsilon \varphi\left(n_{1}\right)+\varepsilon^{2} \varphi\left(n_{2}\right)+\frac{\varepsilon^{3}}{1-\varepsilon} \max _{n_{2} / 2<j \leqslant n_{2}} h_{j}
$$

и т.д.

Существуют две возможности: либо для некоторого $n_{k}>N$

$$
\max _{n_{k} / 2<j \leqslant n_{k}} h_{j}=h_{n_{k}} ;
$$

либо для некоторого $k=k_{0}$ выполняется неравенство $n_{k}<N$. Рассмотрим первую возможность. 
Заметим, прежде всего, что $n_{k}>n / 2^{k}$. Используя представление Караматы для $l(n) / L^{2}(n)$, находим

$$
\frac{\varphi\left(n_{j}\right)}{\varphi(n)}=n \frac{a_{n_{j}}}{n_{j} a_{n}} \exp \left\{-\int_{n_{j}}^{n} \frac{\varepsilon(u)}{u} d u\right\}
$$

Очевидно, что

$$
\left|\int_{n_{j}}^{n} \frac{\varepsilon(u)}{u} d u\right|<\max _{n_{j}<u<n}|\varepsilon(u)| \ln \frac{n}{n_{j}}<j \eta \ln 2, \quad \eta=\max _{u>N}|\varepsilon(u)| .
$$

Следовательно, существует $\varepsilon_{0}$ такое, что при $\varepsilon<\varepsilon_{0}$

$$
\varepsilon^{j} \varphi\left(n_{j}\right)<\varepsilon^{j} \varphi(n) \exp \{j \eta \ln 2\}<\varepsilon^{j / 2} \varphi(n) .
$$

В результате получаем, что при $\varepsilon<\varepsilon_{0}$

$$
h_{n}<\sum_{j=0}^{k-1} \varepsilon^{j} \varphi\left(n_{j}\right)+\frac{\varepsilon^{k}}{1-\varepsilon} \varphi\left(n_{k}\right)<\left(\sum_{j=0}^{k-1} \varepsilon^{j / 2}+\frac{\varepsilon^{k / 2}}{1-\varepsilon}\right) \varphi(n)<\frac{\varphi(n)}{1-\varepsilon^{1 / 2}} .
$$

Во втором случае рекуррентный процесс останавливается при $k=k_{0}=\min \left\{k: n_{k}<\right.$ $N\}$. В результате мы приходим к оценке

$$
h_{n}<\frac{\varphi(n)}{1-\varepsilon^{1 / 2}}+\frac{\varepsilon^{k_{0}-1}}{1-\varepsilon} \max _{k} h_{k}
$$

Поскольку $n_{k}>n / 2^{k}$, то $k_{0}>\log _{2}(n / N)$. Это означает, что $\varepsilon^{k_{0}}<\exp \left\{2^{-1} \ln \varepsilon \log _{2} n\right\}$ при $n>N^{2}$. Следовательно, при достаточно малом $\varepsilon$

$$
\varepsilon^{k_{0}}=o\left(n^{-2}\right)=o(\varphi(n)) .
$$

Из (3.4) и (3.5) следует, что $h_{n}<2 \varphi(n)$ при $n>N^{2}$, если $\varepsilon$ достаточно мало. Возвращаясь теперь к (3.1) и учитывая лемму 2.1 , находим, что при $n \rightarrow \infty$

$$
h_{n}=\frac{l(n)}{n L^{2}(n)}(1+o(1))+O\left(\frac{l^{2}(n)}{n L^{3}(n)}\right)=\frac{l(n)}{n L^{2}(n)}(1+o(1)),
$$

что эквивалентно утверждению теоремы.

4. Дополнение. В настоящем пункте выводится соотношение (1.6) и строится пример, показывающий, что условие $\mathbf{P}(X \geqslant n)=L(n)$, где $L(n)$ - медленно меняющаяся функция, не является достаточным для (1.8).

Лемма 4.1. Пусть выполнено условие (1.3) и, кроме того, существует $n_{0}$ такое, ито $p_{n+1} \leqslant p_{n}$ для $n \geqslant n_{0}$. Тогда

$$
p_{n} \sim \frac{L(n)}{\alpha n^{1+\alpha}}
$$

Д о к а з а т е л ь с т в о. Очевидно, что $\sum_{n \leqslant k \leqslant n(1+\varepsilon)} p_{k} \leqslant n \varepsilon p_{n}$, если $n \geqslant n_{0}$. С другой стороны, ввиду (1.3) для любого $\varepsilon>0$

$$
\sum_{n \leqslant k \leqslant n(1+\varepsilon)} p_{k}>(1-\varepsilon) \frac{L(n)}{n^{\alpha}}\left(1-\frac{1}{(1+\varepsilon)^{\alpha}}\right)
$$

если $n \geqslant n(\varepsilon)>0$.

Таким образом, при $n \geqslant n(\varepsilon)$

$$
p_{n}>\frac{1-\varepsilon}{\varepsilon} \frac{L(n)}{n^{\alpha}}\left(1-\frac{1}{(1+\varepsilon)^{\alpha}}\right) .
$$


Отсюда, поскольку $\lim _{\varepsilon \rightarrow 0} \varepsilon^{-\alpha}\left(1-(1+\varepsilon)^{-\alpha}\right)=\alpha$, следует, что

$$
\liminf _{n \rightarrow \infty} n^{1+\alpha} L^{-1}(n) p_{n} \geqslant \alpha .
$$

Далее, если $(1-\varepsilon) n \geqslant n_{0}$, то

$$
\sum_{n(1-\varepsilon) \leqslant k \leqslant n} p_{k} \geqslant n \varepsilon p_{n} .
$$

С другой стороны, вследствие (1.3) для любого $\varepsilon>0$

$$
\sum_{n(1-\varepsilon) \leqslant k \leqslant n} p_{k}<(1+\varepsilon) \frac{L(n)}{n^{\alpha}}\left(\frac{1}{(1-\varepsilon)^{\alpha}}-1\right),
$$

если $n \geqslant n(\varepsilon)$. В результате имеем для достаточно больших $n$

$$
p_{n}<\frac{1+\varepsilon}{\varepsilon} \frac{L(n)}{n^{1+\alpha}}\left(\frac{1}{(1-\varepsilon)^{\alpha}}-1\right) .
$$

Так как $\lim _{\varepsilon \rightarrow 0}\left(1-(1-\varepsilon)^{\alpha}\right)=\alpha$, то

$$
\limsup _{n \rightarrow \infty} n^{1+\alpha} L^{-1}(n) p_{n} \leqslant \alpha .
$$

Из (4.1) и (4.2) следует утверждение леммы.

П р и м е р 4.1. Пусть $F_{1}(x)$ - любая функция распределения, сосредоточенная на множестве целых неотрицательных чисел, такая, что $1-F_{1}(x)=L(x)$, где $L(x)$ медленно меняется. Положим $y_{k}=L^{-1}\left(2^{-k}\right)$, где $L^{-1}(x)$ - обратная к $L(x)$ функция. Обозначим $x_{k}=\left[y_{k}\right]$. Пусть $F_{2}(\cdot)$ - дискретная функция распределения, сосредоточенная на множестве $\left\{x_{k}\right\}_{1}^{\infty}$, со скачками величины $L^{2}\left(y_{k}\right)$. Очевидно, что $L^{2}\left(y_{k}\right)=4^{-k}$. Поэтому

$$
1-F_{2}\left(x_{k}\right)=\sum_{j=k}^{\infty} 4^{-j}=3^{-1} \cdot 4^{1-k}=\frac{4}{3} L^{2}\left(y_{k}\right) \sim \frac{4}{3} L^{2}\left(x_{k}\right) .
$$

Это означает, что

$$
1-F_{2}(x)=o\left(1-F_{1}(x)\right) .
$$

Далее, пусть $F(x)=2^{-1}\left(F_{1}(x)+F_{2}(x)\right)$. Ввиду (4.3) при $x \rightarrow \infty$

$$
1-F(x) \sim \frac{1}{2} L(x) .
$$

Далее,

$$
F\left(x_{k}+\right)-F\left(x_{k}\right) \geqslant \frac{1}{2}\left(F_{2}\left(x_{k}+\right)-F\left(x_{k}\right)\right)=\frac{1}{2} L^{2}\left(y_{k}\right) .
$$

Пусть случайная величина $X$ имеет распределение $F$. В силу (4.4) и (4.5)

$$
\limsup _{k \rightarrow \infty} \frac{\mathbf{P}\left(X=x_{k}\right)}{\left[\mathbf{P}\left(X \geqslant x_{k}\right)\right]^{2}} \geqslant 1
$$

С другой стороны, в данном случае $\lim _{n \rightarrow \infty} h_{n}=0$, и, следовательно, соотношение (1.8) не может выполняться.

\section{СПИСОК ЛИТЕРАТУРЫ}

1. Колмогоров А.Н. Цепи Маркова со счетным числом возможных состояний. Бюлл. МГУ, секц. А, матем. мех., 1937, т. 1, в. 3, с. 1-16. 
2. Erdös P., Feller W., Pollard H. A property of power series with positive coefficients. Bull. Amer. Math. Soc., 1949, v. 55, p. 201-204.

3. Феллер В. Введение в теорию вероятностей и ее приложения, т. 1. М.: Мир, 1964, $498 \mathrm{c.}$

4. Феллер В. Введение в теорию вероятностей и ее приложения, т. 2. М.: Мир, 1967, $752 \mathrm{c}$.

5. de Bruijn N. G., Erdös P. On a recursion formula and some Tauberian theorems. J. Res. Nat. Bur. Stand., 1953, v. 50, p. 161-164.

6. Garsia A., Lamperti J. A discrete renewal theorem with infinite mean. - Comment. Math. Helv., 1963, v. 37, p. 221-234.

7. Williamson J. A. Random walks and Riesz kernels. - Pacific J. Math., 1968, v. 25, № 2, p. 393-415.

8. Рвачева Е. Л. Об области притяжения многомерных распределений. - Ученые записки Львовского гос. ун-та, 1954, сер. мех.-матем., т. 6, № 29, p. 5-44.

9. Darling D. A. The influence of the maximal term in the addition of independent random variables. - Trans. Amer. Math. Soc., 1952, v. 73, № 1, p. 95-107.

10. Нагаев С.В., Вахтель В.И. О суммах независимых случайных величин без степенных моментов. - Сиб. матем. журн., 2008, т. 49, № 6, с. 1369-1380.

Поступила в редакцию 12.VII. 2010

(c) 2011 г.

MAZURKIEWICZ G.*

\section{ON R $\mathbf{R}^{+}$-WEAKLY STABLE DISTRIBUTION}

Случайный вектор $\mathbf{X}$ называется $\left(\mathbf{R}^{+}\right.$-)слабо устойчивым, если для любых (неотрицательных) случайных величин $\Theta_{1}$ и $\Theta_{2}$, независимых от $\mathbf{X}, \mathbf{X}^{\prime}$ существует (неотрицательная) случайная величина $\Theta$, независимая от $\mathbf{X}$, такая, что

$$
\Theta_{1} \mathbf{X}+\Theta_{2} \mathbf{X}^{\prime} \stackrel{d}{=} \Theta \mathbf{X}
$$

В настоящей статье, отвечая на открытый вопрос, поставленный в (Studia Math., 2005, v. 167, № 3, p. 195-213), мы показываем, что $\mathbf{R}^{+}$слабо устойчивые распределения имеют те же свойства и ту же стохастическую структуру, что и слабо устойчивые распределения.

Ключевые слова и фразы: слабо устойчивое распределение, устойчивое распределение, $\ell_{\alpha}$-симметричное распределение, масштабная смесь, сократимые случайные векторы.

1. Introduction. Throughout the paper we denote by $\mathscr{L}(\mathbf{X})$ the distribution of the random vector $\mathbf{X}$. If random vectors $\mathbf{X}$ and $\mathbf{Y}$ have the same distribution, then we write $\mathbf{X} \stackrel{d}{=} \mathbf{Y}$.

Let $\mathbb{E}$ be a separable Banach space. By $\mathscr{P}(\mathbb{E})$ we denote the set of all probability measures on $\mathbb{E}$. We use the simplified notation $\mathbf{R}^{+}=[0, \infty), \mathscr{P}^{+}=\mathscr{P}([0, \infty)), \mathscr{P}=$ $\mathscr{P}(\mathbf{R})$.

For every $a \in \mathbf{R}$ and every probability measure $\mu$ we define the rescaling operator $T_{a}: \mathscr{P}(\mathbb{E}) \rightarrow \mathscr{P}(\mathbb{E})$ by the formula

$$
T_{a} \mu(A)= \begin{cases}\mu(A / a) & \text { for } a \neq 0 \\ \delta_{0}(A) & \text { for } a=0\end{cases}
$$

* Faculty of Mathematics, Computer Science and Econometrics, University of Zielona Góra, ul. prof. Z. Szafrana 4a, 65-516 Zielona Góra, Poland; e-mail: G.Mazurkiewicz@wmie.uz.zgora.pl 